\title{
Subglottic metastasis of adenoid cystic carcinoma from an anterior tangue primary
}

\begin{abstract}
A 69-year-old lady, whose clinical course included previous pulmonary and cutaneous metastases, presented six years subsequent to primary excision and radiotherapy for left anterior tongue adenoid cystic carcinoma. Computed tomography neck, chest, and abdomen performed for pulmonary metastatic monitoring, incidentally detected a subglottic soft tissue swelling. Microlaryngoscopy and carbon dioxide laser excision completely removed this subglottic polypoidal mass. Histology confirmed respiratory-type mucosa invaded and ulcerated by adenoid cystic carcinoma with perineural invasion. Adenoid cystic carcinoma is locally invasive and only accounts for $1 \%$ of all head and neck malignancies. Hematogenous spread to lung, bone and liver are common, conversely regional lymph node metastases are rare. Despite this, due to the proximity of the primary and metastasis, the complex lymphatic connection between these sites may play a crucial role in this case.
\end{abstract}

Volume 8 Issue 2 - 2017

\author{
Richard Heyes,' Ramkishan Balakumar,' Paul \\ J Tadrous, ${ }^{2}$ Taran Tatla' \\ 'Department of Otolaryngology Head and Neck Surgery, UK \\ 2Department of Cellular Pathology, UK
}

\begin{abstract}
Correspondence: Richard Heyes Department of Otolaryngology Head and Neck Surgery Northwick Park Hospital London, UK, Email r.heyes@hotmail.com
\end{abstract}

Received: March 30, 2017| Published: July 24, 2017

Keywords: adenoid cystic carcinoma, tongue, metastasis, larynx subglottic

Abbreviations: ACC, Adenoid cystic carcinoma; STIR, Short T1-Inversion Recovery

\section{Introduction}

Adenoid cystic carcinoma (ACC) is a well-documented malignant tumor of the major and minor salivary glands that can also originate from other areas of the aero-digestive tract, including the larynx and trachea, and accounts for $1 \%$ of all head and neck malignancies. ACC is locally invasive, with radical surgical resection and postoperative radiotherapy regarded as the treatment of choice. Although regional lymph node metastases are rare, hematogenous spread to lung, bone, and liver are common. ${ }^{1}$ This report describes a unique case of a delayed subglottic metastasis of ACC of the left anterior tongue subsequent to surgical resection and radiotherapy.

\section{Case report}

A 69-year-old lady presented with a constant burning sensation in her tongue of three months' duration. She was found to have an extremely nodular left tongue, especially at the tip and lateral surface, which was exquisitely tender upon palpation. Diagnosis was uncertain, thus MRI was performed, demonstrating a $2.2 \mathrm{~cm}$ by $1.7 \mathrm{~cm}$ lobulated left-side tongue mass of high signal intensity on short T1-inversion recovery (STIR) images (Figure 1). It did not extend into the floor of the mouth and there was no abnormal adenopathy. A biopsy was performed, providing a histological diagnosis of ACC. Staging CT chest and abdomen with contrast showed a non-specific solitary $9.5 \mathrm{~cm}$ pulmonary nodule peripherally at the left lung base with no mediastinal or hilar adenopathy. It was elected in the head and neck cancer multidisciplinary team meeting that she have local excision of the tumor. A partial glossectomy with resection of tumor was performed utilizing a carbon dioxide laser (10watts continuous). During the procedure the ducts were avoided and preserved.

The specimen revealed tongue mucosa and underlying muscle invaded by a grade $2 \mathrm{ACC}, 3.5 \mathrm{~cm}$ in maximum extent by microscopy (but only $1.7 \mathrm{~cm}$ by macroscopic inspection). This discrepancy between macroscopic and microscopic size is most likely due to the highly infiltrative nature of the carcinoma, whereby thin invasive spokes emanate beyond the edge of the main carcinoma mass, often following nerves (Figure 2). The ACC involved the deep margin focally including via extensive perineural invasion. The other resection margins appeared clear and there was no vascular invasion. Surgical resection was followed by postoperative radical radiotherapy (64 Gy in 32 fractions) over six-and-a-half weeks. A right lower lobe lung nodule was removed by wedge excision 18 months after surgery, and a left-sided Video Assisted Thoracoscopic Surgery with lung metastasectomy was performed three years postoperatively. Five years after primary surgery, she had a nodule removed from the vertex of her scalp, which too was metastatic ACC.

A follow-up PET scan was undertaken following scalp lesion excision, showing widespread metastatic disease in bone (primarily lumbar spine) and lung, and MRI highlighted a right fundal lesion (orbit), as well as right occipital and cerebellar metastases. These were treated with palliative radiotherapy (20 Gy in 5 fractions). Repeat CT neck, thorax, and abdomen was subsequently performed, demonstrating a mild reduction of metastatic disease within the occipital lobe, cerebellum and right orbit. However, a new finding of a polypoidal soft tissue thickening in the trachea at the level of the cricoid was also noted (Figure 3). The otolaryngologically asymptomatic patient therefore received clinical review in the head and neck clinic, where flexible nasal laryngoscopy revealed an obvious polypoidal swelling in the right subglottis. A laryngotracheal lesion had not been encountered in previous imaging studies, nor during previous procedures or clinical examinations, thus were the clinical features consistent with metastatic ACC.

Microlaryngoscopy and laser resection of the mass was performed using carbon dioxide laser (10watts- $1 \mathrm{~mm}$ superpulsed), by which time over 6years had passed since primary tumor resection. The subglottic lesion was completely excised and the histology report described the tissue as respiratory-type mucosa invaded and ulcerated by ACC with perineural invasion. On postoperative clinic evaluation, the patient reported no voice complaints and no tumor remnant was visible on flexible nasal laryngoscopic evaluation of the larynx. Ten months following microlaryngoscopic laser resection, the patient was clinically stable following completion of widespread palliative radiotherapy, with no new metastases detected, and without otolaryngological symptoms. DiscussionAdenoid cystic carcinoma 
most commonly arises in the parotid, submandibular and minor salivary glands. ${ }^{1}$ However, it can also occur in other sites, including the tongue which accounts for $4.4 \%$ of cases. ${ }^{2}$ This therefore establishes the uncommon nature of the left-sided ACC tongue mass in this case.

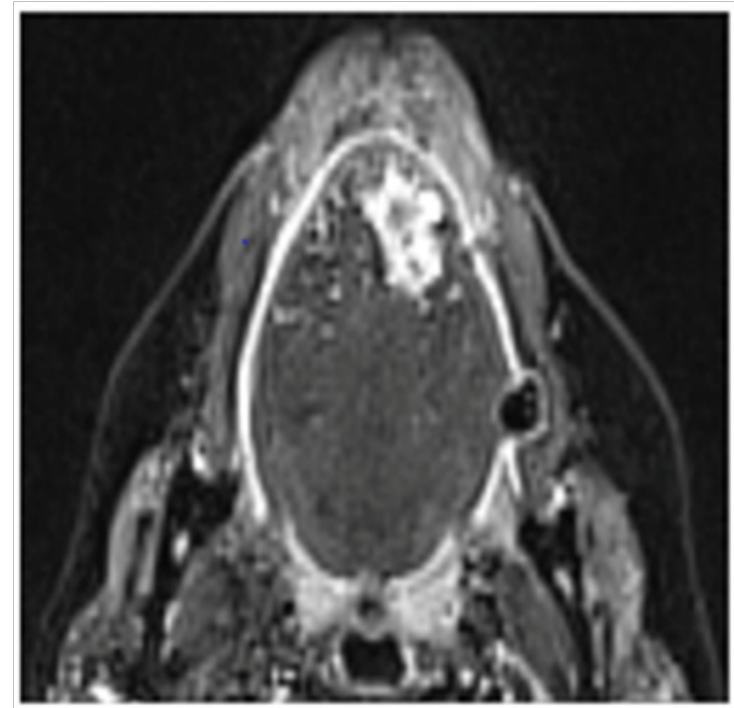

Figure I Axial MRI Scan Showing a $2.2 \mathrm{Cm}$ by $1.7 \mathrm{Cm}$ Lobulated Left-Side Tongue Mass of High Signal Intensity on STIR Images Which Slightly Crosses the Midline Anteriorly.

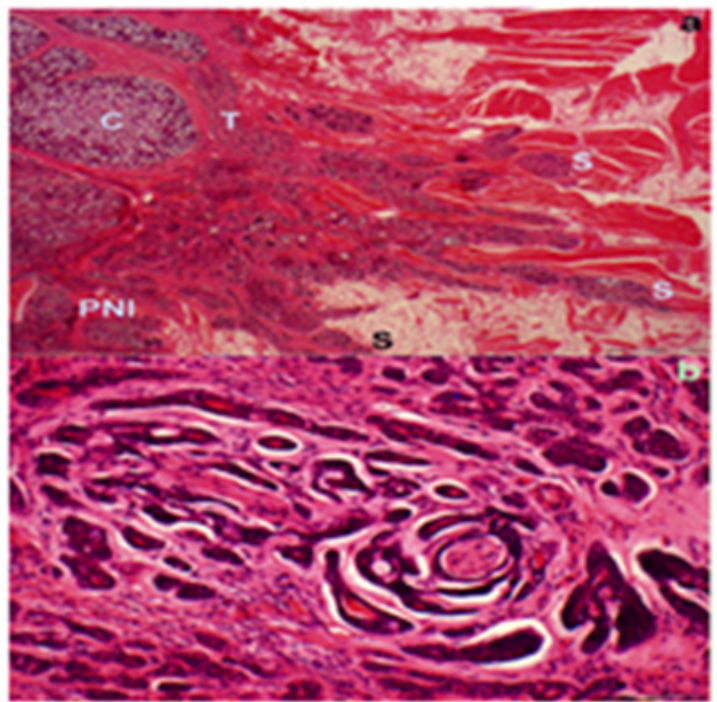

Figure 2 Histopathology of the Resected Primary Tumor in the Tongue. Fig.2a Low Power View (Scale Bar $=$ I Mm) Showing The Main Bulk Of The Tumor On The Left-Side Of The Image Being Predominantly Of Cribriform 'C' And Tubular ' $T$ ' Morphology Without Solid Areas. This Shows Obvious Per neura Invasion 'PNI' Bottom Left And Several Slender Invasive Spokes 'S' Going Out Into The Surrounding Muscle And Adipose. Fig.2b Is a High PowerView (Scale $B a r=100$ Micrometers) of the per neural Invasion Focus Seen in fig.2a. This Also Highlights the Tubular Architecture And Intermediate Cytological Atypia Which Help To Classify This As A Grade 2 Tumor.

Prognosis depends on the histological grade of the carcinoma with grade 1 tumor having a 15 -year survival of about $39 \%$, for grade 2 the 15 -year survival is about $26 \%$, and for grade 3 it is about $5 \%{ }^{3}$ The poor prognosis of ACC is also linked to failure to control distant disease. ${ }^{1}$ This is evident in this case, since despite active treatment of the primary tumor, numerous distant metastases occurred. Among this distant disease, the finding of a subglottic metastasis from the tongue was a highly unusual discovery. Furthermore, the metastasis appeared 6years after primary excision of the tumor. A database search of PubMed, Embase and Cochrane Library was therefore performed with MeSH terms 'metastatic carcinoma; larynx; subglottic AND tongue; adenoid cystic carcinoma; metastasis' to identify any previously published cases. The literature search revealed no cases associated with metastasis to the subglottis from the tongue.

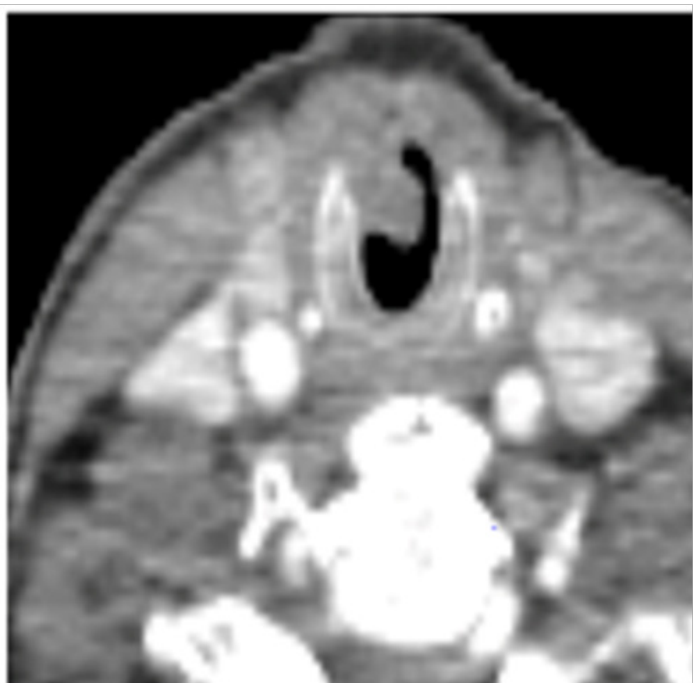

Figure 3 Axial CT scan showing a Polypoidal Soft Tissue Thickening in the Trachea at the Level of the Cricoid.

Metastatic tumors to the larynx are rare and account for 0.1 to $0.4 \%$ of all laryngeal tumors. ${ }^{4}$ The most common primary sites are cutaneous melanoma and renal cell carcinoma, with colorectal adenocarcinoma metastasis increasingly reported. The signs and symptoms of metastatic laryngeal lesions are similar to those of primary laryngeal malignancies, including hoarseness, stridor and dyspnea. Tracheostomy can be performed in those with respiratory distress where the tumor is compromising their airway, but in our case, the patient was asymptomatic allowing this to be avoided. ${ }^{4}$ The mechanism of spread from tongue to subglottis in this case is difficult to identify, but there are various routes by which this could have occurred. Direct translocation of malignant cells from the primary tumor to the larynx by iatrogenic spread may have occurred, but this is unlikely due to the time between resection and metastasis. Furthermore, the strong association of lung metastasis in those who have laryngeal metastasis could also suggest a possible route of spread (i.e. lung to larynx). There is a suggestion that tumor cells implant onto the subglottic area from expectorated neoplastic cells when metastatic or primary lesions exist in the lungs, though insufficient evidence exists to support this. ${ }^{5}$

The hematogenous metastatic route is well-established and explains distant metastasis to lung, bone and brain. ${ }^{6}$ However, the route of spread to the subglottis is less clear. It is known that the tongue empties into either the lingual or common facial vein, through the vena comitans which eventually drain into the internal jugular vein. In dissemination, ACC could potentially reach the subglottis through the cricothyroid branch of the superior laryngeal artery which runs through the cricothyroid ligament, providing a dense capillary network ${ }^{7}$. In a study by Muller et al., the chemokine receptor CXCR4 is highly expressed in ACC. ${ }^{8}$ The liver and lung (organs commonly affected by ACC dissemination) have an abundant expression of 
CXCL12, which is the only known CXCR4 ligand. Therefore, CXCR4 may play a role in hematogenous tumor migration and metastasis of ACC cells. ${ }^{8}$ There is no published evidence to suggest CXCL12 expression specifically in the subglottic region and the limited data on subglottic metastasis makes this difficult to ascertain. Furthermore, the lack of vascular invasion in the resected tongue tumor suggests that the mechanism was not solely by a hematogenous route. There is a correlation with hematogenous and lymphatic spread. There has been a suggestion that tumors unable to intravasate blood vessels directly, spread through lymphatics initially. This may be due to lymphatic capillaries lacking the tight inter-endothelial junctions, smooth muscle cells and basement membranes seen in blood vessels. The fluid transport system of the lymphatics also provides a passive, low-shear system, unlike within blood vessels. ${ }^{6}$ In this particular case, this could be achieved by lymphatic drainage into the left thoracic duct or right lymphatic duct, allowing entry thereon into the venous circulation. ${ }^{6}$

Given that perineural invasion (a common feature of ACC) is most often due to infiltration of perineurial lymphatic spaces, there a remains also the possibility of a direct lymphatic route that connects the tongue to the larynx. This begins from the lingual lymph which is drained through the floor of the mouth and flows mainly dorsally towards the submandibular space. However, single collecting vessels drain the posterior floor of the mouth to the oropharynx, which in turn is connected to the superficial and deep lymphatic networks of the laryngeal mucosa in an uninterrupted manner. The supraglottis has a high density of lymph collectors and may communicate with the subglottis. ${ }^{9}$ This complex system could explain the rare spread of ACC from tongue to the subglottis.

\section{Conclusion}

To the best of our knowledge this is the first documented case of a subglottic metastasis from a tongue cancer. The mechanism by which this has occurred is uncertain but there are various routes by which this could happen. The lymphatic supply from the tongue to the larynx may play a crucial role due to the proximity of the two regions, yet due the patient's multiple metastatic deposits, a hematogenous means is also implicated.

\section{Financial disclosures and support}

None.

\section{Acknowledgments}

None.

\section{Conflicts of interest}

Author declares there are no conflicts of interest.

\section{Funding}

None.

\section{References}

1. Coca-Pelaz A, Rodrigo JP, Bradley PJ, et al. Adenoid cystic carcinoma of the head and neck-An update. Oral Oncol. 2015;51(7):652-661.

2. Feng H, Wang J, Guo P, et al. C3 Vertebral metastases from tongue adenoid cystic carcinoma: A rare case report. Medicine (Baltimore). 2015;94(27):e1135.

3. Fletcher CM. Diagnostic Histopathology of Tumors, (2nd edn). Churchill Livingstone, UK. 2000. p.2296.

4. Zenga J, Mehrad M, Bradley JP. Metastatic Cancer to the Larynx: A Case Report and Update. J Voice. 2016;30(6):774-e779.

5. Puxeddu R, Pelagatti CL, Ambu R. Colon adenocarcinoma metastatic to the larynx. Eur Arch Otorhinolaryngol. 1997;254(7):353-355.

6. Wong SY, Hynes RO. Lymphatic or hematogenous dissemination: How does a metastatic tumor cell decide? Cell Cycle. 2006;5(8):812-817.

7. Kutta H, Steven P, Paulsen F. Anatomical definition of the subglottic region. Cells Tissues Organs. 2006;184(3-4):205-214.

8. Muller A, Sonkoly E, Eulert C, et al. Chemokine receptors in head and neck cancer: association with metastatic spread and regulation during chemotherapy. Int J Cancer. 2006;118(9):2147-2157.

9. Werner JA, Dünne AA, Myers JN. Functional anatomy of the lymphatic drainage system of the upper aerodigestive tract and its role in metastasis of squamous cell carcinoma. Head Neck. 2003;25(4):322-32. 